\title{
Elastic-Stiffness Coefficients of Titanium Diboride
}

Volume 114

\section{Hassel Ledbetter}

Mechanical Engineering

University of Colorado

Boulder, Colorado 80309

\section{Takaho Tanaka}

National Institute for Materials Science,

Sengen, Namiki, Tsukuba, Ibaraki 305-0047

Japan

Number 6

Using resonance ultrasound spectroscopy, we measured the monocrystal elasticstiffness coefficients, the Voigt $C_{\mathrm{ij}}$, of $\mathrm{TiB}_{2}$. With hexagonal symmetry, $\mathrm{TiB}_{2}$ exhibits five independent $C_{i j}: C_{11}, C_{33}$, $C_{44}, C_{12}, C_{13}$. Using Voigt-Reuss-Hill averaging, we converted these monocrystal values to quasiisotropic (polycrystal) elastic stiffnesses. Briefly, we comment on effects of voids. From the $C_{\mathrm{ij}}$, we calculated the Debye characteristic temperature, the Grüneisen parameter, and various sound velocities. Our study resolves the enormous differences between two previous reports of $\mathrm{TiB}_{2}$ 's $C_{\mathrm{ij}}$.
Key words: Debye temperature; elastic constants; Grüneisen parameter; hexagonal crystal; monocrystal; sound velocities; titanium diboride; voids

Accepted: September 10, 2008

Available online: http://www.nist.gov/jres

hassel.ledbetter@colorado.edu

tanaka.takaho@nims.go.jp

\section{Introduction}

Currently, we see a renascence of research on transition-metal diborides. For example, well-known are many current studies on $\mathrm{ReB}_{2}$ and $\mathrm{OsB}_{2}$ as superhard materials, rivaling even diamond.

Titanium diboride's physical and mechanical properties received review elsewhere $[1,2]$. This compound is known well for low mass density, high hardness, high melting point, low electrical resistivity, good thermal conductivity, and good chemical inertness. However, the problems in preparing full-dense, high-quality monocrystals preclude their extensive study. Akimitsu and colleagues [3] reported superconductivity at $40 \mathrm{~K}$ in a same-crystal-structure companion compound: $\mathrm{MgB}_{2}$.

The importance of elastic-stiffness coefficients for both science and technology also received review [4].
Titanium diboride's monocrystal elastic stiffnesses appeared in two reports. The values of Gilman and Roberts [5] depart strongly from the more recent report of Spoor and colleagues [6], mainly in the off-diagonal $C_{12}$ and $C_{13}$. The first measurements were made by a pulse-echo method, the second by resonance ultrasonic spectroscopy, but by a very different mechanical setup than used in the present study.

Here, we report a third set of measurements, which agree closely with the Spoor et al. results. From our $C_{\mathrm{ij}}$ measurements, we estimate the Debye characteristic temperature, the quintessential harmonic-lattice property. Also, we estimate the Grüneisen parameter, the quintessential anharmonic property.

Figure 1 shows the $\mathrm{TiB}_{2}$ crystal structure, obviously a layered structure suggesting strong elastic anisotropy, not observed as shown below, creating a conundrum. 


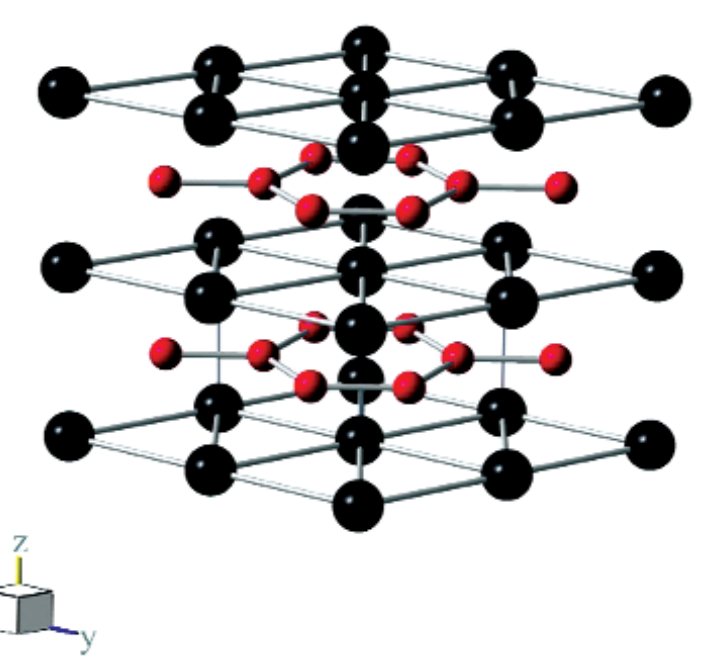

Fig. 1. Schematic crystal structure of titanium diboride $\left(\mathrm{AlB}_{2}\right.$ type, C32, hexagonal, P6/mmm, hP3, $\mathrm{M}=1, \mathrm{D}_{6 \mathrm{~h}}{ }^{1}$, No. 191). Small spheres represent boron atoms, large spheres titanium atoms. The hexagonal boron net resembles strongly that of graphitic carbon; thus we expect strong interatomic bonding within the boron net. The titanium atoms nest in interstices provided by the boron net. The axial ratio equals $3.228 / 3.028=1.066$, relatively high for the $\mathrm{AlB}_{2}$-compound group. Each Ti atom is surrounded by twelve equidistant boron atoms. Each boron atom has three boron atoms at a short distance, and six titanium atoms at a much longer distance.

\section{Measurements}

\subsection{Crystal}

An oriented parallelepiped specimen $1.8 \mathrm{~mm} \times$ $2.3 \mathrm{~mm} \times 3.9 \mathrm{~mm}$ was prepared from a larger crystal grown by a floating zone method $[7,8]$. Fluorescent $\mathrm{x}$-ray analysis revealed no significant impurities. The exact stoichiometry was $\mathrm{TiB}_{1.97}$. The crystal faces with respect to the above dimensions were $[2-1-10]$, [ -1100$]$, [0001]. These directions represent an $a$-axis, the $c$-axis, and the direction orthogonal to both. Laue $\mathrm{x}$-ray diffraction confirmed these orientations within $1^{\circ}$.

\subsection{Method}

To measure the $C_{\mathrm{ij}}$, we used resonance ultrasound spectroscopy, summarized in Fig. 2 [9-11]. Briefly, one clamps lightly a regular-shape (cube, cylinder, cube, parallelepiped) specimen between two piezoelectric transducers. One transducer is swept through frequency and the second transducer detects the specimen's macroscopic vibration frequencies (Fig. 3). Frequencies of a specimen are determined by five factors: (1) shape, (2) size, (3) mass or mass density, (4) elastic-stiffness coefficients $C_{\mathrm{ij}}$, and (5) crystal-axis orientation relative to macroscopic shape. Thus, by

\section{RUS Block Diagram}

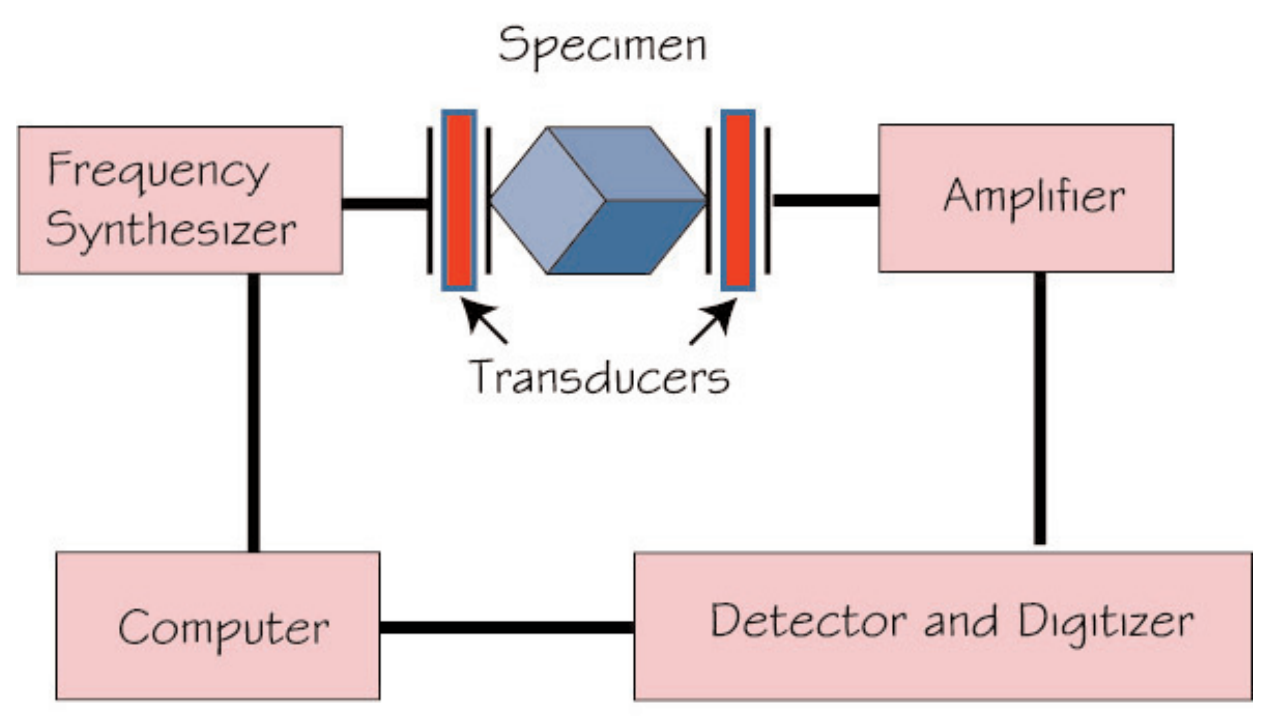

Fig. 2. Schematic measurement setup. Specimen (parallelepiped) is clamped loosely between two piezoelectric transducers. One transducer is swept through frequency. The second transducer detects macroscopic resonance frequencies, which depend on specimen shape, size, mass, and elastic-stiffness coefficients, the $C_{\mathrm{ij}}$. Courtesy of A. Migliori (Los Alamos National Laboratory). 


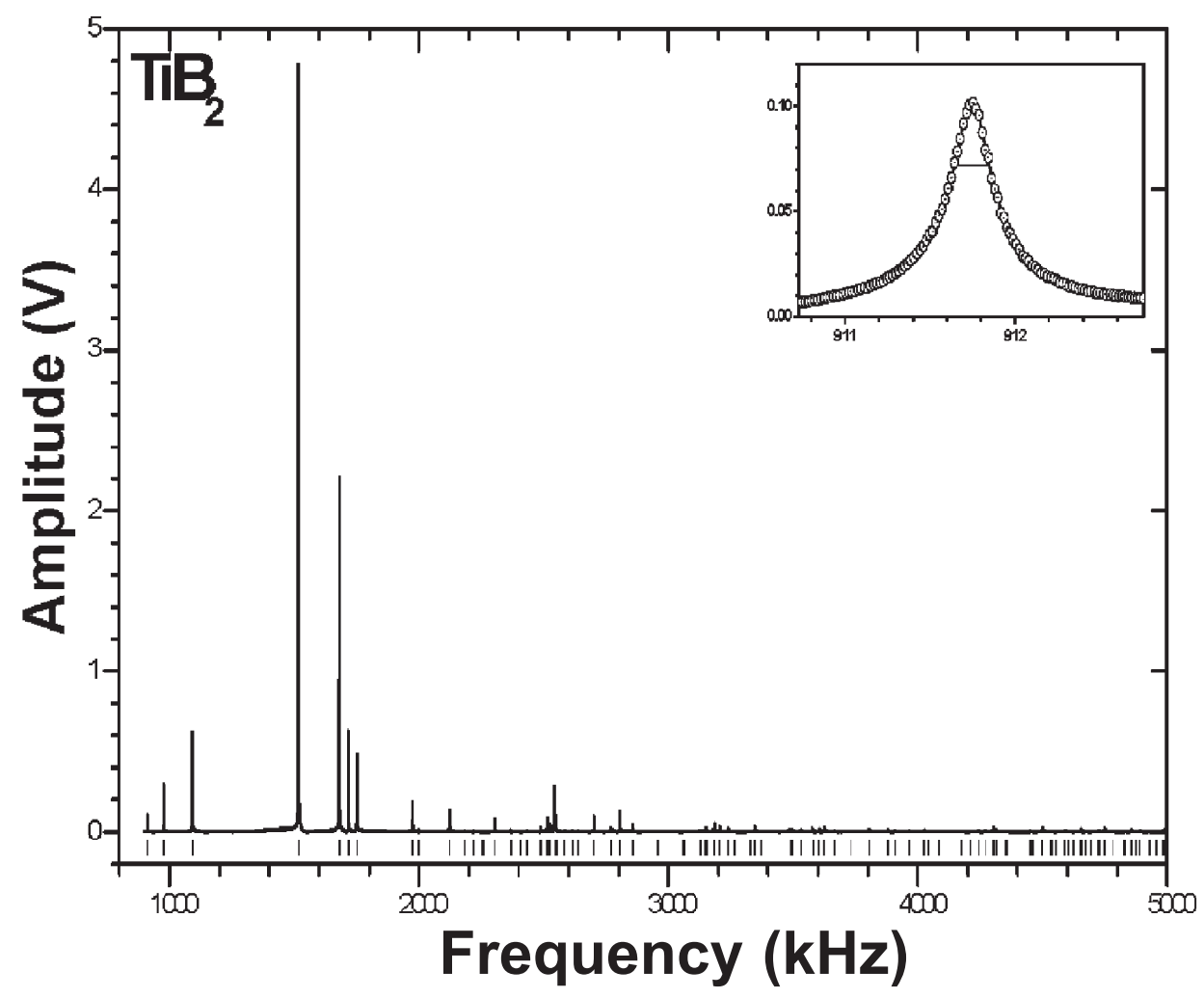

Fig. 3. Macroscopic resonance spectrum. Resonance frequnecies $f_{\mathrm{n}}$ yield $C_{\mathrm{ij}}$ by an inverse-problem calculation. Highly overdetermined, the problem uses about one hundred resonance frequencies $f_{\mathrm{n}}$ to determine the five independent $C_{\mathrm{ij}}$. Vertical bars at bottom show predicted resonance frequencies. The inset shows a resonance-peak profile, with a Lorentzian shape, the half-power width giving the internal friction $Q_{\mathrm{ij}}^{-1}$, the imaginary part of the total $C_{\mathrm{ij}}$.

measuring the resonance frequencies $f_{\mathrm{n}}$, one can determine by an inverse calculation the stiffnesses $C_{\mathrm{ij}}$. The problem is strongly overdetermined: about one hundred $f_{\mathrm{n}}$ to determine five $C_{\mathrm{ij}}$. Not reported here, one can also determine the complete internal-friction tensor $Q_{\mathrm{ij}}^{-1}=\Delta f_{\mathrm{n}} / f_{\mathrm{n}}=C_{\mathrm{ij}}^{*} / C_{\mathrm{ij}}$, where $\Delta f_{\mathrm{n}}$ denotes resonancepeak width, $C_{\mathrm{ij}}{ }^{*}$ the imaginary part, and $C_{\mathrm{ij}}$ the real part of the total $\widetilde{C}_{\mathrm{ij}}$ tensor. Well-described elsewhere [12], the inverse problem involves Lagrangean minimization, the Rayleigh-Ritz method, and a least-squares procedure for measured and deduced $f_{\mathrm{n}}$ values.

We determined mass density by careful mass and size measurements: $\rho=4.502 \pm 0.016 \mathrm{~g} / \mathrm{cm}^{3}$. This compares with reported x-ray mass densities of 4.504 to 4.53 . We ascribe the differences to heavier impurities on the Ti sites. From this mass density, we concluded our specimen contains no significant void content. From the sharp resonance peaks (Fig. 3), we concluded our specimen contains few cracks.

\subsection{Errors}

Errors arise from many sources: crystal orientation, crystal dimensions, nonparallelism, the inverse problem (measured-frequency to $C_{\mathrm{ijk} 1}$ conversion), specimen-transducer interactions. Several authors described these errors elsewhere, especially the first reference [13-15]. The effect of the slight (1\%) departure from stoichiometry is hard to estimate. Because of the strong covalent bonding within the boron planes, we conjecture a very small error arising from an occasional missing boron atom.

\section{Results and Discussion}

Table 1 shows our principal results: the $C_{\mathrm{ij}}$ and their uncertainties. (Because the two previous reports omitted error estimates, very detailed comparisons are precluded.) Only five $C_{\mathrm{ij}}$ are independent because 
Table 1. Monocrystal and polycrystal elastic constants of titanium diboride. Unless specified, all units are GPa, except for the Poisson ratios, $v$, which are dimensionless

\begin{tabular}{|c|c|c|c|}
\hline \multicolumn{2}{|c|}{ Present } & Gilman - Roberts & \multirow[t]{2}{*}{ Spoor et al. ${ }^{\mathrm{b}}$} \\
\hline$\rho\left(\mathrm{g} / \mathrm{cm}^{3}\right)$ & $4.502 \pm 0.016$ & & \\
\hline \multicolumn{4}{|c|}{ Monocrystal elastic constants } \\
\hline$C_{11}$ & $654.4 \pm 1.9$ & 690 & 660 \\
\hline$C_{33}$ & $458.1 \pm 1.4$ & 440 & 432 \\
\hline$C_{44}$ & $262.6 \pm 0.3$ & 250 & 260 \\
\hline$C_{66}$ & $302.7 \pm 0.7$ & 140 & 306 \\
\hline$C_{12}$ & $48.98 \pm 1.4$ & 410 & 48 \\
\hline$C_{13}$ & $95.25 \pm 0.55$ & 320 & 93 \\
\hline$E_{11}$ & $633.3 \pm 2.2$ & 389 & 639 \\
\hline$E_{33}$ & $432.3 \pm 1.7$ & 254 & 408 \\
\hline$v_{12}$ & $0.0460 \pm 0.0022$ & 0.3877 & 0.0437 \\
\hline$v_{13}$ & $0.1984 \pm 0.0015$ & 0.4453 & 0.2059 \\
\hline$v_{31}$ & $0.1354 \pm 0.0010$ & 0.2909 & 0.1314 \\
\hline \multicolumn{4}{|c|}{ Debye characteristic temperature } \\
\hline$\Theta_{\mathrm{D}}(\mathrm{K})$ & $1217 \pm 6$ & 989 & 1211 \\
\hline
\end{tabular}

Voigt-Reuss-Hill-average quasiisotropic (polycrystal) elastic constants

\begin{tabular}{llll}
\hline$C_{\mathrm{L}}$ & 599.9 & 642 & 593 \\
$B$ & 247.5 & 417 & 244 \\
$G$ & 264.3 & 169 & 262 \\
$E$ & 584.7 & 446 & 579 \\
$v$ & 0.1063 & 0.3219 & 0.1037 \\
\hline
\end{tabular}

\section{${ }^{\mathrm{a}}$ Ref. 5 .}

${ }^{\mathrm{b}}$ Ref. 6 .

$C_{66}=\left(C_{11}-C_{12}\right) / 2$. As usual (they fail to correspond directly to a phonon), the off-diagonal $C_{\mathrm{ij}}\left(C_{12}\right.$ and $\left.C_{13}\right)$ show the largest uncertainties. Table 1 also gives the Spoor et al. results [6], which differ from ours by an average of $2.2 \%$. The average uncertainty in our six $C_{\mathrm{ij}}$ is $0.7 \%$. The close agreement between our results and the Spoor et al. results suggests strongly that the earlier Gilman-Roberts results [5] are wrong, the largest discrepancies occurring in the off-diagonal $C_{\mathrm{ij}}: C_{12}$ and $C_{13}$. Further support for the correctness of the Ledbetter-Tanaka/Spoor et al. results arises from $a b$ initio calculations that yielded $B=251 \mathrm{GPa}$ and $v=0.12$ [16], versus 417 and 0.32 for Gilman-Roberts.
Table 1 also shows the principal Young moduli $E_{\mathrm{ii}}$ computed by

$$
E_{\mathrm{ij}}=S_{\mathrm{ij}}^{-1} .
$$

Here $S_{\mathrm{ij}}$ denotes the elastic-compliance tensor, the tensor inverse of the $C_{\mathrm{ij}}$. As we expect from $C_{11}>C_{33}, E_{11}$ exceeds $E_{33}$; that is, $\mathrm{TiB}_{2}$ is much stiffer within the basal plane than along the $c$-axis. Obviously, this relates to the crystal structure where the covalentbonded boron atoms lie in the plane perpendicular to $x_{3}$.

Table 1 shows also the three principal Poisson ratios $v_{\mathrm{ij}}$ computed by

$$
v_{\mathrm{ij}}=-S_{\mathrm{ij}} / S_{\mathrm{ii}} .
$$

Within the boron plane, the Poisson ratio $v_{12}$ is extremely low, reflecting strong covalent bonding and the strong resistance of boron atoms to change their bond angles. The $v_{13}$ Poisson ratio is only slightly below normal, indicating weaker bonds out of the boron-atom planes than those within the planes.

The shear elastic anisotropy of hexagonal crystals can be expressed in various ways. The simplest is $C_{66} / C_{44}, 1.15$ for $\mathrm{TiB}_{2}$, thus weak elastic anisotropy (the isotropic case corresponding to 1.00). Because the Young modulus depends so strongly on the shear modulus, a Young-modulus variation with direction also serves as an effective shear-anisotropy indicator. Spoor and colleagues showed a Young-modulus representation surface; it is nearly spherical [6]. From the alternating-layer boron-titanium crystal structure (Fig. 1), one expects higher elastic anisotropy than one finds.

The lower part of Table 1 gives the averagedover-direction quasiisotropic elastic constants obtained from the $C_{\mathrm{ij}}$ by a Voigt-Reuss-Hill average [17]. These constants include longitudinal modulus $C_{\mathrm{L}}$, bulk modulus $B$, shear modulus $G$, Young modulus $E$, and Poisson ratio $v$. These are the elastic constants appropriate to a full-density nontextured polycrystalline aggregate in which the grain boundaries cause no softening. Grain size produces no effect on elastic constants if it is small relative to specimen size. (A reviewer pointed out that nanosize grains may soften the elastic constants.) The most unusual feature of our results is the high $G / B$ ratio, thus low Poisson ratio. Our nonpublished results on a $\mathrm{YB}_{66}$ monocrystal gave an averaged-over-direction (Kröner method) Poisson ratio of 0.13 . For boron, a handbook value is $v=0.089$ [18]. Both the bulk and shear moduli of $\mathrm{TiB}_{2}$ 
exceed considerably (by $30 \%$ to $40 \%$ ) the handbook values of boron: $248 / 179=1.39$ and $264 / 203=1.30$, showing the strong interatomic bonds in $\mathrm{TiB}_{2}$, both for extension-compression and for shear-bending. Beside implying a low Poisson ratio, the very high $G / B$ ratio holds implications for several other crystal-bonding properties: covalency, Cauchy-relation departure, many-body forces, and others. We plan to discuss all these elsewhere.

The elastic stiffnesses yield three useful sound velocities: the longitudinal velocity

$$
v_{1}=\left(C_{\mathrm{L}} / \rho\right)^{1 / 2}=\left[\frac{B+(4 / 3) G}{\rho}\right]^{1 / 2},
$$

the shear or transverse velocity

$$
v_{\mathrm{s}}=\left(\frac{G}{\rho}\right)^{1 / 2},
$$

and the mean velocity (as defined by Debye)

$$
\frac{3}{v_{\mathrm{m}}^{3}}=\frac{2}{v_{\mathrm{t}}^{3}}+\frac{1}{v_{1}^{3}} \text {. }
$$

We found $v_{1}=1.54, v_{\mathrm{s}}=0.766$, and $v_{\mathrm{m}}=0.835 \mathrm{~cm} / \mu \mathrm{s}$.

From the $C_{\mathrm{ij}}$ and the mass density, we can compute the acoustic Debye characterisitic temperature $\Theta_{D}$. At zero temperature, the acoustic $\Theta_{\mathrm{D}}$ becomes identical with the calorimetric $\Theta_{\mathrm{D}}$ [19]. $\Theta_{\mathrm{D}}$ is proportional to the mean sound velocity:

$$
\Theta_{\mathrm{D}}=K v_{\mathrm{m}}
$$

where $v_{\mathrm{m}}$ denotes mean sound velocity and $\mathrm{K}$ is given by

$$
K=\frac{h}{k}\left(\frac{3}{4 \pi V_{\mathrm{a}}}\right)^{1 / 3}
$$

Here $h$ denotes Planck's constant, $k$ Boltzmann's constant, and $V_{\mathrm{a}}$ atomic volume. The velocity $v_{\mathrm{m}}$ comes from the integration over all directions:

$$
3 v_{\mathrm{m}}^{-3}=\int \sum_{\alpha=1,3} v_{\alpha}^{-3} d \Omega / 4 \pi
$$

Here $v_{1}$ denotes the quasilongitudinal wave velocity, $v_{2}$ and $v_{3}$ the quasitransverse wave velocities, and $d \Omega$ an increment of solid angle. Equation (8) can not be integrated analytically, and numerous numerical and approximate methods have been used for its solution. Phase velocities $v_{a}$ are roots of the Christoffel equations:

$$
\begin{aligned}
& \operatorname{det}\left(C_{\mathrm{ijkl}} x_{\mathrm{j}} x_{\mathrm{k}}-\rho v^{2} \delta_{\mathrm{il}}\right)=0 . \\
& \text { (sum over repeated indices) }
\end{aligned}
$$

This expression follows from equations of motion for plane, monochromatic waves, where $\rho$ denotes mass density, $C_{\mathrm{ijkl}}$ fourth-rank elastic-stiffness tensor, $x_{\mathrm{i}}$ components of the unit wave vector relative to the cubic axes, and $\delta_{\text {il }}$ the Kronecker operator. Equation (9) usually yields three distinct real roots $\rho v^{2}$.

We computed an exact $\Theta_{\mathrm{D}}$ by using a distribution of 489 vectors proposed by Overton and Schuch [20], which we distributed over the usual 48 [100]-[110][111] stereographic unit triangles, thus a total of 23472 directions. We obtained $\Theta_{\mathrm{D}}=1217 \mathrm{~K}$. This result differs enormously from the handbook calorimetric value, $1576 \mathrm{~K}$. Often, calorimetric values contain large errors because of large extrapolations to zero temperature and/or large uncertainties in the lowest-temperature lattice specific heat.

From $B$ and from handbook values of heat capacity $C$, volume thermal expansivity $\beta$, and volume $V$, we computed the effective Grüneisen parameter:

$$
\gamma=B_{\mathrm{S}} \beta V / C_{\mathrm{P}}
$$

We obtained $\gamma=1.71$, the handbook values for boron and titanium being 1.85 and 1.33 . Alternative gammas can be computed when one knows the third-order elastic-stiffness coefficients, the $C_{\mathrm{ijklmn}}[21]$. The few gammas known for compounds similar to $\mathrm{TiB}_{2}$ preclude any comparisons. Following Pearson's reasoning [22], the Ti-B bonds would lead to $\gamma=2$.

Finally, because some authors suspect voids/cracks in $\mathrm{TiB}_{2}$, we want to describe briefly how the above fulldense quasiisotropic elastic constants would change with voids. Focusing on $\mathrm{Al}_{2} \mathrm{O}_{3}$, Ledbetter, Lei, and Datta [23] gave a theory for void effects on elastic constants. Principal results include the following: Voids soften the bulk modulus more than the shear modulus. In the dilute limit, for spherical voids, 
our results agree with the classical results of Mackenzie [24]:

$$
\begin{aligned}
& \frac{\Delta B}{B}=-3 c, \quad\left(v_{0}=1 / 3\right) \\
& \frac{\Delta G}{G}=-\frac{15}{8} c . \quad\left(v_{0}=1 / 3\right)
\end{aligned}
$$

Here, $c$ denotes void volume fraction and $v_{0}$ the void-free-state Poisson ratio. However, we emphasize that the often-used rule-of-thumb that elastic stiffness varies as mass density is true only in the dilute limit. Void shape plays a key role, especially if the voids possess an oblate-spheroid (disc) shape. Dunn and Ledbetter [25] focused on the interesting, unexpected effects of voids and cracks on the Poisson ratio. Other authors addressed this problem using various approximations [26].

\section{Conclusions}

1. Our elastic-stiffness-coefficient measurements on titanium diboride support the report of Spoor and colleagues rather than the older (now handbook) values of Gilman and Roberts. The principal differences in the two previous $C_{\mathrm{ij}}$ sets lie in $C_{12}$ and $C_{13}$.

2. From the $C_{\mathrm{ij}}$ we computed several additional useful physical properties:

(i) sound velocities; (ii) Debye temperature;

(iii) Grüneisen parameter.

3. Our computed acoustic Debye temperature, $1217 \mathrm{~K}$, is about $20 \%$ lower than the handbook calorimetric value.

4. Our computed Grüneisen parameter, 1.71, suggests the importance of Ti-B bonds along with B-B bonds.

5. Using the $C_{66} / C_{44}$ ratio as a shear-mode elasticanisotropy criterion, $\mathrm{TiB}_{2}$ shows low shearmode anisotropy, 1.15. A surprise because of the B-Ti-B ... layer crystal structure.

6. Our elastic constants estimated for a full-dense polycrystal depart strongly from those given in Munro's review [2], especially in the uncertainties. For example, Munro proposed a $70 \%$ uncertainty in the Poisson ratio and a $24 \%$ uncertainty in the bulk modulus. Our polycrystal results differ from the Spoor et al. result by only $2 \%$. This finding agrees with the well-known fact that modern measurement methods give the elastic-stiffness coefficients easily within one percent.

7. Finally, we are surprised by the low elastic shear anisotropy shown by such a strongly layered crystal structure. Reference [8] gives a possible explanation. Part of the boron-atom $p$-obitals lie in a $p$ - $d$ hybridized band, weakening the $p$-electron contribution to B-B bonding. If the bonding weakens, the anisotropy decreases.

\section{Acknowledgments}

Thanks especially to Dr. Edwin Fuller (NISTGaithersburg) for an incisive critical reading. S. Kim (NIST-Boulder) helped with measurements. Most of this study proceeded while $\mathrm{H}$. Ledbetter was at NIST-Boulder. F. Drymiotis (Clemson U., Physics) contributed Fig. 1.

\section{References}

[1] J. Hoard and R. Huges, Chemistry of Boron and its Compounds (Wiley, New York, 1967).

[2] R. Munro, Material properties of titanium diboride, J. Res. Natl. Inst. Stand. Technol. 105, 709-720 (2000).

[3] J. Nagamatsu, N. Kakagawa, T. Muranaka, Y. Zenitani, and J. Akimitsu, Superdonductivity at $39 \mathrm{~K}$ in magnesium diboride, Nature 410, 63-64 (2001).

[4] H. Ledbetter, Elastic properties, in Materials at Low Temperatures (Amer. Soc. Metals, Metals Park, Ohio, 1983) pp. 1-45.

[5] J. Gilman and B. Roberts, Elastic constants of $\mathrm{TiC}$ and $\mathrm{TiB}_{2}$, J. Appl. Phys. 32, 1405 (1961).

[6] P. Spoor, J. Maynard, M. Pan, D. Green, J. Hellmann, and T. Tanaka, Elastic constants and crystal anisotropy of titanium diboride, Appl. Phys. Lett. 70, 1959-1961 (1997).

[7] Y. Ishizawa and T. Tanaka, in Science of Hard Materials, E. A. Almond, C. A. Brooks, and R. Warren, eds. (Adam Hilger, Boston 1986) pp. 29-43.

[8] T. Tanaka and Y. Ishizawa, The deHaas-van Alphen effect in $\mathrm{TiB}_{2}$, J. Phys. C 13, 6671-6676 (1980).

[9] O. Anderson, Rectangular parallelepiped resonance-A technique of resonance ultrasound and its applications to the determination of elasticity at high temperatures, J. Acoust. Soc. Amer. 91, 2245-2253 (1982).

[10] A. Migliori and J. Sarrao, Resonant Ultrasound Spectroscopy (Wiley-Interscience, New York, 1997).

[11] J. Maynard, Resonant ultrasound spectroscopy, Phys. Today 49 (1), 26-31 (1996).

[12] P. Heyliger, A. Jilani, H. Ledbetter, R. Leisure, and C.-L. Wang, Elastic constants of isotropic cylinders using resonant ultrasound, J. Acoust. Soc. Amer. 94, 1482-1487 (1993). 
[13] P. Spoor, P. White, and J. Maynard, An investigation of computational problems associated with resonant ultrasound spectroscopy, J. Acoust. Soc. Am. 99, 2492-2603 (1996).

[14] R. Leisure and F. Willis, Resonant ultrasound spectroscopy, J. Phys.: Condens. Matter 9, 6001-6029 (1997).

[15] R. Schwarz and J. Vuorinen, Resonant ultrasound spectroscopy: applications, current status, and limitations, J. Alloys and Compounds 310, 243-250 (2000).

[16] K. Panda and K. Chandran, Determination of elastic constants of titanium diboride $\left(\mathrm{TiB}_{2}\right)$ from first principles using FLAPW implementation of the density-functional theory, Computational Mater. Sci. 35, 134-150 (2006).

17] H. Ledbetter, Monocrystal-polycrystal elastic-constant models, in Handbook of Elastic Properties of Solids, Liquids, and Gases, Volume III (Academic, New York 2000) pp. 313-324.

[18] K. Gschneidner, Physical properties and interrelationship of metallic and semimetallic elements, Solid State Phys. 16, 275426 (1964).

[19] G. Leibfried and W. Ludwig, Theory of anharmonic effects in crystals, Solid State Phys. 12, 275-444 (1961).

[20] W. Overton and A. Schuch, Method for highly accurate numerical spatial integration: Applications to lattice dynamics of cubic-type crystals, Los Alamos Lab. Rept. 3162 (1965).

[21] H. Ledbetter and S. Kim, Elastic Grüneisen parameters of cubic elements and compounds, in Handbook of Elastic Properties of Solids, Liquids, and Gases, Volume II (Academic, New York, 2001) pp. 107-122.

[22] R. Pearson, Chemical Hardness (Wiley, New York, 1992) p. 188.

[23] H. Ledbetter, M. Lei, and S. Datta, Elastic constants of porous ceramics, in Handbook of Elastic Properties of Solids, Liquids, and Gases, Volume III (Academic, New York, 2001) pp. 463471.

[24] J. Mackenzie, Elastic constants of a solid containing spherical holes, Proc. Phys. Soc. Lond. B63, 2-11 (1950).

[25] M. Dunn and H. Ledbetter, Poisson ratio of porous and microcracked solids, J. Mater. Res. 10, 2715-2722 (1995).

[26] M. Kachanov, On the effective elastic constants of cracked solids, Int. J. Fract. 146, 295-399 (2007).

About the authors: Hassel Ledbetter is a materials scientist in Mechanical Engineering at the University of Colorado in Boulder, Colorado. He retired from the Materials Reliability Division at NIST-Boulder, where he established the world's most complete elasticcoefficient-measurement laboratory, especially for measurements to liquid-helium temperature. Then he spent four years at Los Alamos National Laboratory, at the National High Magnetic Field Laboratory, studying low-temperature physical properties of various materials, including actinides such as plutonium. Takaho Tanaka is a materials scientist at the National Institute for Materials Science (NIMS). He retired from the Boride Research Group at NIMS, where he established the world's highest-temperature crystal-growth laboratory, especially for growing crystals of refractory materials such as rare-earth borides, transition metal borides, and carbides. He also discovered and characterized many icosahedral boron-rich rare-earth borides.

The National Institute of Standards and Technology is an agency of the U.S. Department of Commerce. 